\title{
TENSÕES ORGANIZACIONAIS COMO IMPULSIONADORAS DE CAPACIDADES DINÂMICAS EM ORGANIZAÇÕES HÍBRIDAS
}

\section{RESUMO}

Desde a teoria estruturalista sabemos que as tensões e conflitos organizacionais não são necessariamente prejudiciais aos propósitos de um determinado empreendimento. Partindo deste pressuposto tivemos nesse artigo teórico o objetivo de analisarmos de que forma a presença de tensões organizacionais pode ser uma potencial impulsionadora de capacidades dinâmicas em organizações híbridas, ilustradas no artigo como negócios sociais. Como principal contribuição teórica do estudo defendemos que tensões de pertencimento, aprendizado, organização e desempenho podem impulsionar capacidades dinâmicas de comportamentos e habilidades, rotinas e processos e mecanismos de aprendizagem e governança do conhecimento em organizações híbridas e potencialmente em outras tipologias organizacionais. Também trazemos um estímulo para que os gestores de organizações híbridas estejam atentos às tensões de seus empreendimentos pois, se por um lado estas podem impulsionar capacidades dinâmicas, por outro, podem levar ao declínio de suas atividades, diante do atual cenário de frequentes mudanças tecnológicas e institucionais que vivemos.

Palavras-chave: Capacidades Dinâmicas; Tensões Organizacionais; Organizações Híbridas; Negócios Sociais.

\section{ORGANIZATIONAL TENSIONS AS DRIVING FORCES OF DYNAMIC CAPABILITIES IN HYBRID ORGANIZATIONS}

\section{ABSTRACT}

Since structuralist theory we know that organizational tensions and conflicts are not necessarily detrimental to the purposes of a given enterprise. Based on this assumption we had in this theoretical article the objective of analyzing how the presence of organizational tensions can be a potential driver of dynamic capacities in hybrid organizations, illustrated in the article as social enterprise. As the main contribution of the study, we stress that the tensions of belonging, learning, organization and performance can boost dynamic capabilities of behaviors and skills, routines and processes and mechanisms of learning and knowledge governance in hybrid organizations and potentially in other organizational typologies. We also bring a stimulus so that the managers of hybrid organizations are aware of the tensions of their enterprises because, while on the one hand these can boost dynamic capabilities, on the other, they can lead to the decline of their activities, given the current scenario of frequent technological changes and institutions we live.

Keywords: Dynamic Capabilities; Organizational Tensions; Hybrid Organizations; Social Enterprises. 
Tensões Organizacionais como Impulsionadoras de Capacidades Dinâmicas em Organizações Híbridas

\section{TENSIONES ORGANIZACIONALES COMO IMPULSIONADORAS DE CAPACIDADES DINÁMICAS EN ORGANIZACIONES HÍBRIDAS}

\section{RESUMEN}

Desde la teoría estructuralista sabemos que las tensiones y conflictos organizacionales no son necesariamente perjudiciales para los propósitos de un determinado emprendimiento. A partir de este presupuesto tuvimos en ese artículo teórico el objetivo de analizar de qué forma la presencia de tensiones organizacionales puede ser una potencial impulsora de capacidades dinámicas en organizaciones híbridas, ilustradas en el artículo como negocios sociales. Como principal contribución teórica del estudio defendemos que las tensiones de pertenencia, aprendizaje, organización y desempeño pueden impulsar capacidades dinámicas de comportamientos y habilidades, rutinas y procesos y mecanismos de aprendizaje y gobernanza del conocimiento en organizaciones híbridas y potencialmente en otras tipologías organizacionales. También traemos un estímulo para que los gestores de organizaciones híbridas estén atentos a las tensiones de sus emprendimientos pues, si por un lado éstas pueden impulsar capacidades dinámicas, por otro, pueden llevar al declive de sus actividades, ante el actual escenario de frecuentes cambios tecnológicos y, institucionales que vivimos.

Palabras clave: Capacidades Dinámicas; Tensiones Organizacionales; Organizaciones Híbridas; Negocios Sociales.

\footnotetext{
${ }^{1}$ Doutoranda em Administração de Empresas pela Escola de Administração de Empresas de São Paulo da Fundação Getulio Vargas - EAESP/FGV. Brasil. E-mail: aline8barbosa@ gmail.com

${ }^{2}$ Doutorando em Administração de Empresas pela Escola de Administração de Empresas de São Paulo da Fundação Getulio Vargas - EAESP/FGV. Professor da Escola Superior de Engenharia e Gestão - ESEG e Professor de Pósgraduação da Fundação Instituto de Administração - FIA. Brasil. E-mail: mromdias @ hotmail.com

${ }^{3}$ Doutorando em Administração de Empresas pela Escola de Administração de Empresas de São Paulo da Fundação Getulio Vargas - EAESP/FGV. Brasil. E-mail: rodrigo.assuncao.r@gmail.com
} 


\section{INTRODUÇÃO}

Para atender a diferentes objetivos, muitas vezes conflitantes entre si, organizações híbridas enfrentam tensões organizacionais. Este é o caso, por exemplo, dos negócios sociais, empreendimentos que ao mesmo tempo devem gerar lucro e perseguir prioritariamente uma missão voltada ao atendimento de demandas sociais (Michelini \& Fiorentino, 2012 Grassl, 2012; Jäger \& Schröer, 2014). Estas tensões acontecem quando estes negócios enfrentam conflitos de metas ou diferentes demandas entre stakeholders, discordâncias entre prioridades, foco em múltiplas metas, ganhos tangíveis - como lucro financeiro - versus ganhos intangíveis - como motivação dos colaboradores e propósitos sociais (Smith \& Lewis, 2011; Grassl, 2012; Smith, Gonin \& Besharov , 2013).

Desde a teoria estruturalista sabemos que as tensões e conflitos organizacionais não são necessariamente prejudiciais aos propósitos de um determinado empreendimento (Bastos \& Seidel, 1992). Entendemos, portanto, que se uma tensão pode ser capaz de trazer benefícios organizacionais, ela também pode ser capaz, em determinados contextos, de trazer capacidades dinâmicas às organizações, sejam estas de comportamentos e habilidades, rotinas e processos e mecanismos de aprendizagem e governança do conhecimento (Meirelles \& Camargo, 2014).

Muito se discute sobre de que forma as organizações podem criar e manter capacidades dinâmicas (Teece, Pisano \& Shuen, 1997), entretanto, o papel das tensões organizacionais não tem sido satisfatoriamente tratado na literatura sobre o tema. Diante desta lacuna, para a construção deste artigo partimos da seguinte questão: Por que a presença de tensões organizacionais é uma potencial impulsionadora de capacidades dinâmicas em organizações híbridas?

Com o intuito de responder a questão de pesquisa proposta, este trabalho teórico trata da relação entre tensões organizacionais vivenciadas por organizações híbridas e capacidades dinâmicas. Com base em levantamento bibliográfico sobre estas temáticas desenvolvemos três proposições que trazem as tensões organizacionais como impulsionadoras de capacidades dinâmicas no contexto de organizações híbridas.

Trazemos neste artigo duas principais contribuições para o campo da pesquisa em estratégia empresarial e mais especificamente para as abordagens teóricas de capacidades dinâmicas, que tem crescido de forma exponencial no Brasil nos últimos anos (Aragão, Forte \& Oliveira, 2010). A primeira reside no fato de abordarmos, de forma inédita, um importante fator que influencia a construção de capacidades dinâmicas pelas organizações, ou seja, as tensões organizacionais. Entendemos que tensões de pertencimento, aprendizado, organização e desempenho podem impulsionar capacidades dinâmicas de comportamentos e habilidades, rotinas e processos ou mecanismos de aprendizagem e governança do conhecimento. A segunda contribuição, esta de cunho prático, é direcionada aos gestores de organizações híbridas, os quais são estimulados por meio de nosso estudo a compreenderem que tensões só podem impulsionar as capacidades dinâmicas de seus empreendimentos se forem bem geridas; caso contrário, estas tensões podem levar as organizações ao declínio de suas atividades, haja vista o atual cenário de frequentes mudanças tecnológicas e institucionais que vivemos (Teece, 2009; Danneels 2011).

Além desta introdução, este artigo está dividido em quatro seções. A primeira delas trata do referencial teórico sobre tensões organizacionais no contexto de organizações híbridas. A segunda seção traz definições teóricas presentes na literatura sobre capacidades dinâmicas e aplicáveis ao objetivo de nosso artigo. Na seção seguinte apresentamos a discussão de como as tensões organizacionais relacionam-se com capacidades dinâmicas em organizações híbridas, discussão esta que é realizada por meio de três proposições e de modelo conceitual sobre o tópico. Por fim, na seção de considerações finais, resgatamos o objetivo de nosso artigo, identificamos os principais resultados e contribuições alcançados e tratamos de oportunidades de pesquisas futuras.

\section{TENSÕES EM ORGANIZAÇÕES HÍBRIDAS}

A tentativa de conciliar internamente demandas concorrentes nas organizações pode levar ao cenário de conflitos internos e confusões, que podem ser expressos na forma de tensões (Battilana \& Dorado, 2010; Jay, 2013; Pache \& Santos, 2013). De acordo com Smith \& Lewis (2011) as tensões organizacionais emergem do comprometimento à estrutura da organização, de sua cultura e de suas práticas e processos e são geradas por existirem demandas competitivas e excludentes entre si, concomitantemente ao fato de gestores serem pressionados a tomarem decisões. Esta situação pode trazer dilemas para as organizações, que terão que ser resolvidos dependendo da natureza e forma de gerenciamento de tais tensões.

Como exemplo temos os negócios sociais, que devido à complexidade no gerenciamento dos conflitos e competição entre lógicas comerciais e sociais - e que por esta razão são denominadas organizações híbridas neste artigo - enfrentam desafios e tensões entre suas características essenciais, ou seja, a missão da empresa voltada ao atendimento e resolução de demandas sociais e a necessária mobilização de recursos financeiros e humanos para a manutenção do empreendimento (Battilana \& Dorado, 2010; Pache \& Santos, 2013). 
Tensões Organizacionais como Impulsionadoras de Capacidades Dinâmicas em Organizações Híbridas

Uma característica essencial deste tipo de empreendimento - a busca pelo atingimento de uma causa social - apresenta inúmeros desafios, como o gerenciamento de múltiplos stakeholders, que possuem objetivos distintos, assim como são distintos também os objetivos do empreendimento. $\mathrm{O}$ acionista ou investidor, caso exista, possui interesse em obter maiores lucros com seu investimento e a comunidade em que a organização está inserida tem, por sua vez, o interesse na resolução de seus problemas e demandas sociais, como as relacionadas ao desenvolvimento da saúde ou educação (Doherty, Haugh \& Lyon, 2014).

A mobilização dos recursos financeiros também gera desafios para este tipo de empreendimento, devido a uma maior necessidade de compreensão do valor social por aqueles que controlam os recursos e a visão de longo prazo sobre resultados sociais e não meramente econômicos (Doherty, Haugh \& Lyon, 2014). O recurso humano também é um fator importante para organizações híbridas, pois existem colaboradores interessados em maximizar seus ganhos e também existem aqueles engajados com o objetivo social do empreendimento. Garantir a motivação destes perfis diferentes de indivíduos, também por meio de recompensas que não sejam meramente monetárias, pode ser uma forma de auxiliar neste desafio (Doherty, Haugh \& Lyon, 2014).

$\mathrm{Na}$ tentativa de compreender os fatores que geram as tensões e possivelmente os dilemas em empresas, outros pesquisadores dedicaram esforços para sistematizar elementos importantes presentes na gestão e cotidiano das organizações, como o estudo de Smith \& Lewis, (2011); Smith, Gonin e Besharov (2013), que classificou as tensões organizacionais em tensões (i) de aprendizado, (ii) de pertencimento, (iii) de organização e (iv) de desempenho, conforme Figura 1:

Figura 1 - Categorização de Tensões Organizacionais

\begin{tabular}{|c|c|c|}
\hline $\begin{array}{l}\text { Aprendizado / Pertencimento } \\
\text { Conflitos entre a necessidade de } \\
\text { adaptação e mudança e o desejo } \\
\text { de reter ou senso ordenado de } \\
\text { auto propósito }\end{array}$ & $\begin{array}{l}\text { Aprendizado } \\
\text { Esforços para ajustar, renovar, } \\
\text { alterar e inovar promove tensões } \\
\text { entre construir e destruir o } \\
\text { passado para criar o futuro }\end{array}$ & $\begin{array}{l}\text { Aprendizado / Organização } \\
\text { Rotina organizacional e } \\
\text { capacidades buscam } \\
\text { estabilidade, clareza, foco, e } \\
\text { eficiência ao mesmo tempo } \\
\text { permitindo resultados } \\
\text { dinâmicos, flexíveis e ágeis }\end{array}$ \\
\hline $\begin{array}{c}\text { Pertencimento } \\
\text { Identidade promove tensões } \\
\text { entre o individual e o coletivo e } \\
\text { entre valores divergentes, } \\
\text { funções e sociedade }\end{array}$ & $\begin{array}{l}\text { Perten cimento/Organização } \\
\text { Tensões entre o coletivo } \\
\text { Construir o o individual, } \\
\text { capacidades individualismo } \\
\text { para o futuro } \quad \text { versus ação } \\
\text { enquanto garante o c coletiva } \\
\text { o sucesso no presente } \\
\text { Aprendizado/Des em penho }\end{array}$ & $\begin{array}{c}\text { Organização } \\
\text { Estrutura e liderança promovem } \\
\text { colaboração e competição, } \\
\text { empoderamento e direção, e } \\
\text { controle e flexibilidade }\end{array}$ \\
\hline $\begin{array}{l}\text { Desempenho / Pertencimento } \\
\text { Conflito entre identificação e } \\
\text { objetivos como atores negociam } \\
\text { identidades individuais com } \\
\text { demandas sociais e ocupacionais }\end{array}$ & $\begin{array}{c}\text { Desempenho } \\
\text { Multiplicidade promove } \\
\text { objetivos múltiplos e } \\
\text { competitivos, como Stakeholders } \\
\text { buscam sucesso organizacional } \\
\text { divergente }\end{array}$ & $\begin{array}{l}\text { Desempenho / Organização } \\
\text { Interação entre meios e fins, } \\
\text { empregados versus demandas de } \\
\text { clientes, alto compromisso } \\
\text { versus alto desempenho }\end{array}$ \\
\hline
\end{tabular}

Fonte: Adaptado de Smith \& Lewis (2011, p. 383). 
No estudo sobre tensões de negócios sociais de Walchhutter, Romani-Dias e Barbosa (2016) discutiuse sobre o caso do Banco Pérola, organização que iniciou sua trajetória oferecendo educação tecnológica e cursos profissionalizantes para jovens, mas que mudou sua trajetória ao perceber que a maioria destes jovens não possuíam acesso aos recursos financeiros em bancos tradicionais e passou a oferecer microcrédito orientado às atividades microempreendedoras deste público. Este processo de mudança gerou (i) tensões de aprendizado no negócio, ao ter que optar por uma nova atividade e novas formas de trabalhar.

Tensões sobre (ii) pertencimento envolvem questões de identidade dos indivíduos e relacionamentos interpessoais, e estão relacionadas aos conflitos entre interesses individuais e coletivos, principalmente devido aos valores divergentes entre pessoas (Smith \& Lewis, 2011; Smith, Gonin \& Besharov, 2013). Este é o caso da Rede Asta, que tem como principal atividade a distribuição de produtos para casa e moda feitos à mão por grupos de artesãos de regiões de baixa renda do Brasil, e que possui grupos de produção diferentes em história, cultura e valores - alguns grupos são mais estruturados e formados por mulheres de classe média e outros são menores e formados por mulheres de comunidade de baixa renda. Desta forma, este empreendimento lida com diferentes estruturas, culturas, processos e práticas que consequentemente geram tensões para conciliar as condições particulares de cada grupo (Iizuka, Varela \& Larroude, 2015).

As organizações em geral também apresentam tensões que estão relacionadas aos seus (iii) processos (de organização) e acontecem entre a necessidade de colaboração e a competição, pois a estrutura e a liderança promovem este cenário. $\mathrm{O}$ perfil de profissionais contratados exerce grande influência sobre os conflitos entre empoderamento de pessoas e direcionamento de funções e também sobre a rotina sistematizada e processos de mudança; como exemplo, alguns indivíduos estarão abertos aos procedimentos mais "engessados" e direcionados, e outros serão mais livres e necessitam de maior autonomia (Smith \& Lewis, 2011; Smith, Gonin \& Besharov, 2013).

Assim como o caso da Rede Asta, que lida com diferentes perfis de profissionais na sua linha de produção, o empreendimento Goóc, produtor de calçados, bolsas e mochilas a partir de matérias primas como pneus reciclados e lonas de caminhão, também enfrenta tensões relacionadas a sua preocupação social e ambiental. Como exemplo, a Goóc possui parte substancial de sua mão de obra com baixa qualificação profissional e considerada de baixa mobilidade social, como ex-presidiários e mulheres acima de 50 anos de idade, fato este gerador de tensões, pois a decisão por esta mão de obra poderia, em uma primeira análise, comprometer os processos e prazos do negócio, ou seja, sua eficiência, eficácia e efetividade (Walchhutter, Romani-Dias \& Barbosa, 2016).

Por fim, (iv) tensões de desempenho ocorrem quando organizações enfrentam conflitos de metas ou diferentes demandas entre seus stakeholders internos e externos. O foco e os objetivos mudam de acordo com a ótica de cada parte interessada no negócio, pois a visão de sucesso e mensuração dos resultados será analisada de formas diferentes (Smith \& Lewis, 2011; Smith, Gonin \& Besharov, 2013). Os exemplos do Banco Pérola, da Rede Asta e da Goóc apresentam tensões de desempenho, pois seus gestores devem optar entre reduzir seus lucros para obterem um maior alcance do impacto social de seu negócio, como é o exemplo da Goóc, ao utilizar no processo fabril matérias primas mais onerosas que as tradicionais, como pneus e lonas reciclados (Iizuka, Varela \& Larroude, 2015; Walchhutter, Romani-Dias \& Barbosa, 2016).

Ao analisarmos a Figura 2 percebemos que além dos quatro tipos de tensões mencionados, ainda existem "mistos" entre elas, como tensões entre desempenho e organização, que ocorrem, por exemplo, na interação entre os interesses dos colaboradores versus as demandas dos clientes; tensões entre aprendizado e desempenho, que ocorrem diante da necessidade de construção de capacidades para o futuro concomitantemente à manutenção do sucesso presente; tensões de aprendizado e pertencimento, que ocorrem devido às demandas de mudanças e o desejo de manter o senso do propósito organizacional (Smith \& Lewis, 2011; Smith, Gonin \& Besharov, 2013).

Além destas tensões mistas, há também as tensões entre organização e aprendizado, que relacionam-se à rotina organizacional e as capacidades de buscar estabilidade, clareza, foco e eficiência, concomitantemente com resultados dinâmicos, flexíveis e ágeis, tensões de pertencimento e desempenho, que estão relacionadas com conflitos entre a identificação e os objetivos dos indivíduos e como estes atores negociam suas identidades individuais com as demandas sociais e ocupacionais e, por fim, as tensões entre pertencimento e organização, que envolvem questões individuais e coletivas, decisões motivadas pelo individualismo ou a importância da ação coletiva (Smith \& Lewis, 2011; Smith, Gonin \& Besharov, 2013).

\section{CAPACIDADES DINÂMICAS}

Com origem no campo de administração estratégia e influenciada principalmente por teorias como a Teoria Schumpeteriana (Schumpeter, 1934), a Teoria da Firma (Penrose, 1959), a Teoria dos Custos de Transação (Williamson, 1975) e a Visão Baseada 
Tensões Organizacionais como Impulsionadoras de Capacidades Dinâmicas em Organizações Híbridas

em Recursos (Barney, 1991), as capacidades dinâmicas buscam responder a clássica questão de como as empresas alcançam e sustentam vantagem competitiva, principalmente em ambientes de rápida mudança (Teece, Pisano \& Shuen, 1997). Diversos estudos têm evidenciado tanto o crescimento quanto a relevância do conceito de capacidades dinâmicas na compreensão de diferentes fenômenos organizacionais em diversas áreas e temas do campo gestão e organizações, como os estudos de Barreto (2010), Peteraf, Stefano e Verona (2013), Vogel e Güttel (2013) e Wilden, Devinney e Dowling (2016).

As frequentes mudanças tecnológicas e institucionais, típicas de um mundo cada vez mais transitório e da rápida mudança, fazem com que as organizações tenham que lidar cada vez mais com a reconfiguração de seu arcabouço de recursos, competências e habilidades em busca do aumento de seus desempenhos, necessitando desenvolver suas capacidades dinâmicas, que segundo a definição clássica de Teece et al. (1997, p. 516) refere-se à "habilidade da firma em integrar, construir e reconfigurar competências internamente e externamente, para endereçar ambientes em rápida mudança." Ao longo do desenvolvimento teórico do conceito, os autores têm se debruçado em diferentes definições, todas elas referentes a questão de mudança organizacional, principalmente às mudanças relacionadas ao seu conjunto de recursos, habilidades, competências, capacidades e rotinas. O Quadro 1 ilustra as diferentes definições dos autores:

\begin{tabular}{|c|c|}
\hline AUTORES & DEFINIÇÃO \\
\hline Teece et al. (1997) & $\begin{array}{l}\text { Habilidade da firma em integrar, construir e reconfigurar competências internamente e } \\
\text { externamente, para endereçar ambientes em rápida mudança. }\end{array}$ \\
\hline Collis (1994) & Capacidade em inovar mais rapidamente ou de forma melhor do que a concorrência. \\
\hline Eisenhardt e Martin (2000) & Processos da firma que usam recursos para corresponder ou criar mudanças de mercado. \\
\hline Zollo \& Winter (2002) & $\begin{array}{l}\text { Padrão aprendido e estável de atividade coletiva, baseado em mecanismos de aprendizagem, } \\
\text { por meio dos quais a organização sistematicamente gera e modifica suas rotinas operacionais } \\
\text { em busca de melhoria na efetividade. }\end{array}$ \\
\hline Winter (2003) & Capacidade para operar, estender, modificar ou criar capacidades comuns. \\
\hline Andreeva \& Chaika (2006) & $\begin{array}{l}\text { Capacidades dinâmicas são aquelas que habilitam a organização a renovar suas competências- } \\
\text { chave conforme ocorrem mudanças no ambiente operacional. }\end{array}$ \\
\hline Helfat et al. (2009) & $\begin{array}{l}\text { Capacidade de uma organização criar, estender ou modificar sua base de recursos } \\
\text { propositadamente. }\end{array}$ \\
\hline Wang \& Ahmed (2007) & $\begin{array}{l}\text { Comportamento constantemente orientado a integrar, reconfigurar, renovar e recriar seus } \\
\text { recursos e capacidades e melhorar e reconstruir as capacidades-chave em resposta às mutações } \\
\text { do ambiente, para atingir e sustentar a vantagem competitiva. }\end{array}$ \\
\hline Bygdas (2006) & $\begin{array}{l}\text { Processo de ativar estruturas distribuídas de conhecimento e redes fragmentadas de } \\
\text { procedimentos e entendimentos soltos que desenvolvem práticas mais eficientes que não são } \\
\text { facilmente imitáveis. }\end{array}$ \\
\hline Dosi et al. (2008) & $\begin{array}{l}\text { Heurísticas gerenciais e as ferramentas de diagnósticos constituem o cerne das capacidades } \\
\text { dinâmicas. }\end{array}$ \\
\hline McKelvie \& Davidson (2009) & $\begin{array}{l}\text { Capacidades dinâmicas como um feixe de outras capacidades (capacidade de geração de } \\
\text { ideias; de introdução de rupturas no mercado; e capacidades de desenvolvimento de novos } \\
\text { produtos, serviços e novos processos). }\end{array}$ \\
\hline Teece $(2007,2009,2012)$ & $\begin{array}{l}\text { Capacidade de sentir o contexto do ambiente; aproveitar oportunidades, gerenciar ameaças e } \\
\text { transformações. }\end{array}$ \\
\hline
\end{tabular}

Quadro 1 - Definições de Capacidades Dinâmicas Fonte: Meirelles e Camargo (2014, p. 51-52) 
Na definição de Meirelles e Camargo (2014) organizações que estão inseridas em ambientes dinâmicos ou também estáveis necessitam desenvolver capacidades e processos de ordem superior que auxiliem na adaptação dinâmica frente os diferentes tipos de mudanças no contexto vivenciado por essas organizações. Sob essa ótica, o foco está no processo pelo qual a empresa desenvolve e renova suas competências, e este processo é condicionado tanto pelas escolhas passadas da própria organização como pelo dinamismo do ambiente.

Sobre este ponto, para a construção desse artigo adotamos a visão de Eisenhardt e Martin (2000), que ao contrário da visão proposta por Teece et al. (1997), argumentam que o foco no dinamismo do ambiente dá lugar a uma ênfase de que as capacidades podem ser aprendidas e duplicadas em várias empresas e diferentes ambientes, e uma empresa que atua em ambientes dinâmicos não necessariamente possuirá capacidades dinâmicas (Peteraf, Di Stefano \& Verona, 2013). Nesse sentido, os fundamentos das capacidades dinâmicas são: a capacidade de criar mudanças no mercado e a capacidade de reação às mudanças externas. Adotamos também a proposta de síntese das Capacidades Dinâmicas de Meirelles e Camargo (2014, p.55-56) que a entendem da seguinte forma:

[...] capacidades dinâmicas como a capacidade que é baseada em decisões deliberadas e recorrentes, composta por processos combinatórios de capacidades que permitem criar, estender, modificar ou reconfigurar as capacidades-chave da organização e sua base de recursos e competências, fazendo uso de três elementos componentes: (a) comportamentos e habilidades; (b) rotinas e processos; (c) mecanismos de aprendizagem e governança do conhecimento.

Os comportamentos estão relacionados com a lealdade e o comprometimento com a mudança (Andreeva \& Chaika, 2006; Teece, 2009), as habilidades de mudança e inovação estão relacionadas com a identificação de oportunidades de mercado, o reconhecimento do valor das informações externas e seu aprendizado para aplicá-las comercialmente (Wang \& Ahmed, 2007). Destacam-se também as habilidades que não necessariamente relacionam-se com a rotina operacional do negócio, como a comunicação, a resolução de conflitos ou o gerenciamento de projetos e de pessoas (Andreeva \& Chaika, 2006; Meirelles \& Camargo, 2014).

Os processos e as rotinas de suporte às capacidades dinâmicas estão relacionados aos padrões de comportamentos que podem ser aprendidos e repetidos por diversos atores (Gerard, 2009). Para Teece (2007), os processos e rotinas incluem desde rotinas de identificação de segmentos de mercado nos quais o negócio deseja atuar até processo para descentralizar as decisões com mudanças nas estruturas organizacionais (Meirelles \& Camargo, 2014).

Por fim, estão os mecanismos de aprendizagem e governança do conhecimento que contemplam processos de integração de know how externo, aprendizagem, compartilhamento e integração de conhecimento. Possuir habilidade para integrar e combinar ativos, incluindo os de conhecimento, é de extrema importância para o desenvolvimento dos processos de melhoria e, de acordo com Zollo e Winter (2002), este processo está relacionado a um ciclo de evolução do conhecimento e faz uso de três mecanismos de aprendizagem: acumulação de experiência, articulação de conhecimento e codificação do conhecimento.

\section{TENSÕES COMO IMPULSIONADORAS DE CAPACIDADES DINÂMICAS}

De acordo com Lüscher \& Lewis (2008) as tensões organizacionais podem servir como um meio para que gerentes considerem outras perspectivas, alterem suas premissas e explorem questões de maneiras diferentes. Como exemplo, entendemos que gestores de organizações híbridas, como os negócios sociais, podem dedicar esforços para entender os tipos de tensões que este hibridismo gera e desenvolver a habilidade de utilizar estas tensões como aprendizado para nutrir e refinar seus recursos internos e externos, preparando-se para reconfigurar estes recursos de acordo com as mudanças no contexto de negócio em que está inserido (Lüscher \& Lewis, 2008; O'Reilly \& Tushman, 2008, Danneels, 2011).

A capacidade dos gestores para aproveitar oportunidades por meio da integração de recursos novos e existentes para superar a estagnação e a dependência do passado é o núcleo das capacidades dinâmicas (O’Reilly \& Tushman, 2008). Estas capacidades devem ser incorporadas nas rotinas, estruturas e processos organizacionais existentes e devem ser encontradas na forma como a organização opera, em suas estruturas e culturas e na mentalidade da liderança sênior (O'Reilly \& Tushman, 2008; Smith et al., 2013).

Para compreender melhor esta relação, os autores Lüscher e Lewis (2008) realizaram uma pesquisa-ação na Empresa Danish Lego com o objetivo de ajudar os gerentes de produção no gerenciamento de tensões de pertencimento, performance e organização que emergiram devido a uma extensa reestruturação. Confrontar estas tensões ajudou os gerentes a sair de ciclos repetidos, pois quando as tensões se espalharam em torno dos relacionamentos elas estimularam a defensiva e a necessidade do confronto. Estas tensões 
Tensões Organizacionais como Impulsionadoras de Capacidades Dinâmicas em Organizações Híbridas

contribuíram para os gerentes considerarem outras perspectivas, alterarem suas premissas e explorarem questões de maneiras fundamentalmente diferentes, impulsionando assim, o desenvolvimento de capacidades dinâmicas (Lüsher \& Lewis, 2008).

Os autores Doherty, Haugh e Lyon (2014), esquematizaram um quadro com os tipos de tensões em organizações híbridas, os trade-offs dessas situações de conflito e exemplos práticos de gerenciamento para os gestores deste tipo de empreendimento para que a capacidade dinâmica possa ser impulsionada. À partir do exemplo das tensões do Quadro 2, podemos perceber que estas tensões podem impulsionar as capacidades dinâmicas, como por exemplo, o desafio em atrair e reter colaboradores com competências adequadas para este tipo de empreendimento, pode gerar uma tensão no processo de seleção, onde haja equilíbrio entre a experiência com aspectos sociais e financeiros. Um exemplo de gerenciamento para este tipo de tensão é a realização de treinamento com foco em questões de cunho social e questões de cunho comercial. Este processo de desenvolvimento de treinamento específico pode ser considerado como uma capacidade dinâmica de mecanismos de aprendizado. Defende-se que esta situação de conflito, ao ser gerenciada, impulsiona o desenvolvimento de uma capacidade dinâmica para o empreendimento. 
Tensões Organizacionais como Impulsionadoras de Capacidades Dinâmicas em Organizações Híbridas

\begin{tabular}{|c|c|c|c|c|}
\hline $\begin{array}{c}\text { Características } \\
\text { distintas }\end{array}$ & Desafios & Tensões & Trade-offs (perda e ganho) & $\begin{array}{c}\text { Impulsionador de Capacidade } \\
\text { Dinâmica }\end{array}$ \\
\hline \multirow{2}{*}{ Missão } & $\begin{array}{l}\text { Alcançar os objetivos de negócio e } \\
\text { sociais }\end{array}$ & $\begin{array}{c}\text { Demandas conflitantes entre as } \\
\text { necessidades de clientes e necessidades de } \\
\text { outras partes interessadas }\end{array}$ & $\begin{array}{l}\text { Sacrificar a criação de valor social } \\
\text { para captura de valor econômico }\end{array}$ & $\begin{array}{c}\text { Usar a missão social como uma força } \\
\text { para a direção estratégica }\end{array}$ \\
\hline & $\begin{array}{l}\text { Gerenciar as demandas de múltiplos } \\
\text { stakeholders e manter a legitimidade }\end{array}$ & $\begin{array}{l}\text { Discordâncias sobre as prioridades } \\
\text { realizada por diferentes grupos }\end{array}$ & $\begin{array}{c}\text { Não buscar a maximização do lucro } \\
\text { propositadamente }\end{array}$ & $\begin{array}{l}\text { Encontrar as melhores condições } \\
\text { sociais onde criação de valor leve à } \\
\text { rentabilidade e vantagem competitiva }\end{array}$ \\
\hline \multirow{4}{*}{$\begin{array}{l}\text { Mobilização } \\
\text { recurso financeiro }\end{array}$} & \multirow{2}{*}{$\begin{array}{l}\text { Negócios Sociais não podem ser } \\
\text { entendidos como viáveis clientes por } \\
\text { instituições financeiras convencionais }\end{array}$} & $\begin{array}{l}\text { A importância relativa de ganho contra } \\
\text { outras receitas }\end{array}$ & \multirow{2}{*}{$\begin{array}{c}\text { Estratégias de preços duplos para } \\
\text { diferentes } \\
\text { grupos de clientes }\end{array}$} & $\begin{array}{l}\text { Utilizar a receita excedente para } \\
\text { reinvestir em missão social }\end{array}$ \\
\hline & & $\begin{array}{c}\text { Questões éticas envolvidas no acesso as } \\
\text { diferentes fontes de renda }\end{array}$ & & $\begin{array}{l}\text { Aproveitar o mix de capital financeiro } \\
\text { tanto causas comerciais e filantrópicas }\end{array}$ \\
\hline & \multirow{2}{*}{$\begin{array}{c}\text { Falta de compreensão do Negócio } \\
\text { Social e valor social por aqueles que } \\
\text { controlam o acesso a recursos } \\
\text { financeiros }\end{array}$} & $\begin{array}{l}\text { Expectativas e demandas conflitantes } \\
\text { entre as diferentes partes interessadas }\end{array}$ & \multirow{2}{*}{$\begin{array}{l}\text { Persuadir investidores a aceitares uma } \\
\text { taxa mais baixa e mais lenta de retorno } \\
\text { em troca de criação de valor social }\end{array}$} & $\begin{array}{l}\text { Acesso mais baixo para os investidores } \\
\text { sociais do que a taxa de mercado }\end{array}$ \\
\hline & & $\begin{array}{c}\text { Operando sob restrições financeiras } \\
\text { devido às recursos financeiros } \\
\text { inadequados }\end{array}$ & & $\begin{array}{c}\text { Novas formas legais para incentivar } \\
\text { investimento }\end{array}$ \\
\hline \multirow{3}{*}{$\begin{array}{l}\text { Mobilização } \\
\text { recurso humano }\end{array}$} & $\begin{array}{c}\text { Recursos financeiros limitados } \\
\text { restringir salários e remunerações em } \\
\text { Negócios Sociais } \\
\end{array}$ & $\begin{array}{l}\text { Gerenciar motivação e recompensas para } \\
\text { funcionários e voluntários }\end{array}$ & $\begin{array}{l}\text { Balancear o pagamento de altos } \\
\text { salários em negócios sociais e investir } \\
\text { na realização de missão social }\end{array}$ & $\begin{array}{c}\begin{array}{c}\text { Equilíbrio entre funcionários membros } \\
\text { da diretoria com habilidade social e } \\
\text { comercial }\end{array} \\
\end{array}$ \\
\hline & $\begin{array}{l}\text { Falta de qualificação e competência } \\
\text { que combinem com objetivos sociais e } \\
\text { financeiros }\end{array}$ & $\begin{array}{l}\text { Voluntários não percebem que precisam } \\
\text { ter habilidades e experiência em algumas } \\
\text { áreas de prestação de serviço }\end{array}$ & $\begin{array}{l}\text { Recrutar voluntários contra a alta } \\
\text { rotatividade de voluntários }\end{array}$ & $\begin{array}{c}\text { Utilização de medidas não-pecuniárias } \\
\text { para motivar e recompensar } \\
\text { funcionários, voluntários e } \\
\text { administradores }\end{array}$ \\
\hline & $\begin{array}{l}\text { Atrair e reter funcionários com } \\
\text { competências adequadas }\end{array}$ & $\begin{array}{l}\text { Processo de seleção de administradores } \\
\text { que proporcione um equilíbrio entre a } \\
\text { experiência com o social e o financeiro }\end{array}$ & $\begin{array}{c}\text { Maiores salários e remunerações em } \\
\text { Negócios Sociais reduzem a } \\
\text { atratividade destes negócios para } \\
\text { doadores, voluntários e outros } \\
\text { stakeholders }\end{array}$ & $\begin{array}{c}\text { Treinamento social e comercial para } \\
\text { administradores e demais stakeholders }\end{array}$ \\
\hline
\end{tabular}

Quadro 2 - Tensões em Organizações Híbridas e Impulsionadores de Capacidades Dinâmicas

Fonte: Adaptado de Doherty, Haugh e Lyon (2014, p. 428). 
Outro exemplo de trabalho que tratou sobre tensões e dilemas de negócios sociais no Brasil, de Iizuka, Varela \& Larroude (2015), organiza alguns tipos de conflitos enfrentados pela Rede Asta, um tipo de organização híbrida que concilia objetivos socioeconômicos, conforme quadro 3:

\begin{tabular}{|c|c|}
\hline $\begin{array}{l}\text { Types of } \\
\text { tensions }\end{array}$ & Facts related to Rede Asta's history and operations \\
\hline Performance & $\begin{array}{l}\text { - Asta attempts to disseminate the concept of fair trade throughout the production chain; } \\
\text { - Financial results alone are not enough to define priorities among sales channels; } \\
\text { - The network\&'s stated target audiences are inconsistent (focusing on women or valuing diversity?). }\end{array}$ \\
\hline Organizations & $\begin{array}{l}\text { - Each group\&'s singular production speed may compromise the delivery schedule for requests of } \\
\text { large quantities of items; } \\
\text { - The network needs to operate with two organizations: an association and a corporation (also } \\
\text { applicable in learning). }\end{array}$ \\
\hline Belonging & $\begin{array}{l}\text { - The Rede Asta cause, based on the triple bottom line, has so far served as the means to create a } \\
\text { feeling of belonging to the organization, especially considering that the expectations of different } \\
\text { internal stakeholders are as yet being met. }\end{array}$ \\
\hline Learning & $\begin{array}{l}\text { - The sales team worked hard for years to combine financial return with their social and } \\
\text { environmental causes: } \\
\text { - Starting in 2011, the network realized the need to strengthen its brand in order to consolidate itself } \\
\text { as social business and has been investing in this purpose; } \\
\text { - Meanwhile, Asta also understood the importance of production scale in order to establish itself in } \\
\text { the market. }\end{array}$ \\
\hline
\end{tabular}

Quadro 3 - Tipos de Tensões na Rede Asta

Fonte: Iizuka, Varela \& Larroude (2015, p. 394).

Um tipo de tensão observada neste estudo, por exemplo, é sobre a velocidade de produção única de cada grupo, que pode comprometer o cronograma de entrega dos pedidos. Este empreendimento, devido sua natureza social, emprega artesãs de baixa renda da comunidade, impactando assim sua linha de produção devido a seleção e contratação de mão de obra não apenas por qualificação profissional, mas considerando aspetos sociais, como a baixa renda destas mulheres.

Caso este negócio estabeleça processos padrões ou recorte a produção respeitando com a especialidade de cada funcionária, as habilidades de cada artesã podem ser exploradas (também por meio da governança do conhecimento) e, consequentemente esse processo pode ser aprendido e replicado para outras artesãs, contribuindo assim para o desenvolvimento da capacidade de rotinas e processos.

\subsection{Proposições para o Desenvolvimento de Capacidades Dinâmicas}

A partir das contribuições apontadas pelos autores Smith, Gonin e Besharov (2013) acerca das tensões organizacionais, e de Meirelles \& Camargo (2014) sobre capacidades dinâmicas, apontamos neste artigo proposições que indicam as tensões organizacionais como impulsionadoras no desenvolvimento das capacidades dinâmicas em organizações híbridas.
As tensões sobre pertencimento, como os conflitos de interesses pessoais e coletivos decorrentes dos valores divergentes entre os funcionários, podem impulsionar a capacidade de comportamento e habilidade de mudança e inovação. A partir do reconhecimento desta tensão o gestor pode, por exemplo, desenvolver trabalhos com os funcionários, pois nesta capacidade, o aspecto-chave é a construção de lealdade e comprometimento dos funcionários frente às mudanças, que pode ser desenvolvida por meio da comunicação aberta, transparente e que permita diálogos francos entre as partes (Andreeva \& Chaika, 2006; Teece, 2009).

Seguindo esta lógica, as tensões de organização, como as que estão relacionadas à necessidade de colaboração e a competição entre os funcionários e as tensões sobre desempenho, como conflitos entre metas ou demandas diferentes entre stakeholders internos $\mathrm{e}$ externos, podem impulsionar as habilidades de mudança e inovação, por meio do desenvolvimento de habilidades que não estejam ligadas às atividades operacionais cotidianas dos indivíduos na organização, como, por exemplo, as habilidades de autoaprendizagem, de comunicação, de negociação, de liderança, de resolução de problemas (Andreeva \& Chaika, 2006). A partir destas relações apresentamos a seguinte proposição (1): 
Proposição 1: As tensões em aprendizado, pertencimento, organização $e$ desempenho em organizações híbridas podem impulsionar o desenvolvimento da capacidade de comportamentos $e$ habilidades de mudança e inovação.

Tensões relacionadas ao aprendizado, como, por exemplo, o aprendizado de novas formas de executar tarefas, podem impulsionar o desenvolvimento de mecanismos de aprendizagem e da governança do conhecimento. O desenvolvimento de múltiplas soluções, a discussão coletiva para articulação do conhecimento, as sessões para esclarecimentos de dúvidas e o acúmulo de experiência e legitimação dos novos conhecimentos gerados são capacidades que a organização híbrida pode desenvolver por meio desta tensão (Zollo \& Winter, 2002).

As tensões sobre pertencimento também podem impulsionar os mecanismos de aprendizado e governança do conhecimento, pois ao estimular a discussão participativa com diversos colaboradores, os gestores dos empreendimentos podem ouvir diversas partes, conhecer perfis distintos e contribuir para que os públicos sintam-se parte do negócio tornando o ambiente favorável a um melhor desempenho (Zollo \& Winter, 2002). Com base nestas argumentações, apresentamos a seguinte proposição (2):

Proposição 2: As tensões em aprendizado, pertencimento, organização e desempenho em organizações híbridas podem impulsionar o desenvolvimento da capacidade de mecanismos de aprendizado e governança do conhecimento.
As tensões relacionadas ao desempenho do negócio, como as diferentes expectativas dos stakeholders, que buscam maior abrangência do impacto social por parte de um grupo e o aumento dos lucros por parte de outro grupo, podem impulsionar o desenvolvimento da capacidade dinâmica relacionada às rotinas e aos processos de identificação de segmentos de mercado alvo, definição de fronteiras do negócio, protocolos de tomadas de decisão e investimento e solução para problemas de agência e grupos internos (Andreeva \& Chaika, 2006; Teece, 2007). Com processos e rotinas bem estabelecidas cada stakeholder pode encontrar espaço para o atendimento de suas expectativas relacionadas ao negócio. A partir destas relações, apresentamos a seguinte proposição (3):

Proposição 3: As tensões em aprendizado, pertencimento, organização e desempenho em organizações híbridas podem impulsionar o desenvolvimento da capacidade de processos e rotinas de busca ou inovação.

Com base nas três proposições defendemos que as tensões de aprendizado, pertencimento, organização e desempenho, presentes em organizações híbridas, podem impulsionar o desenvolvimento de capacidades dinâmicas como comportamentos, habilidades, rotinas, processos, mecanismos de aprendizagem e governança do conhecimento, voltados para mudança e inovação. A partir das proposições deste estudo apresentamos na Figura 2 o modelo conceitual que trata da relação entre as categorias de tensões em organizações híbridas e os elementos componentes de capacidades dinâmicas: 
Figura 2 - Modelo de Tensões Organizacionais como Impulsionadoras de Capacidades Dinâmicas

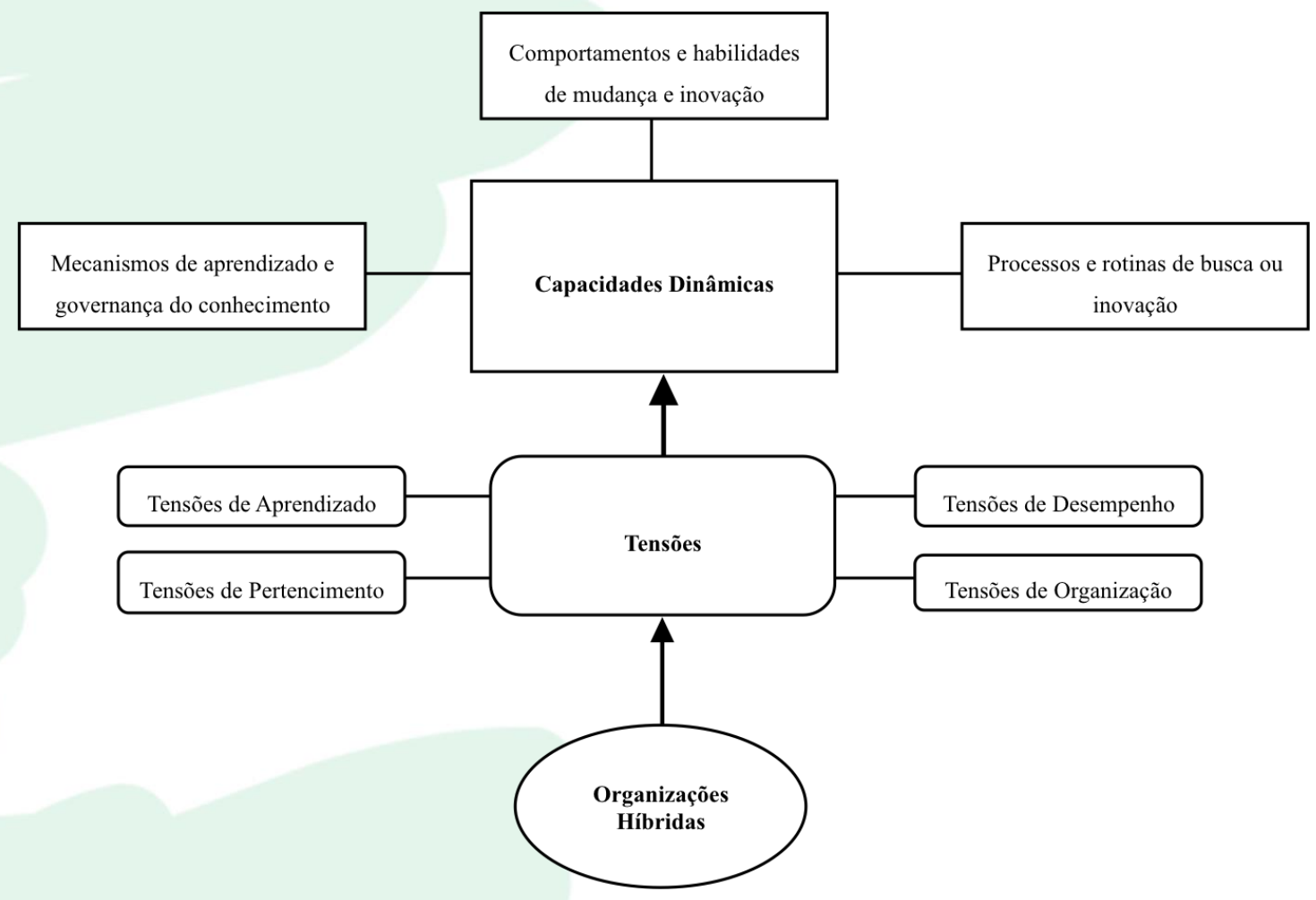

Fonte: Baseado em Smith \& Lewis (2011) e Meirelles \& Camargo (2014).

O modelo apresentado ilustra as proposições feitas, propondo que as tensões de (i) aprendizado (conhecimento); pertencimento (identidade/relacionamentos interpessoais); (iii) organização (processos) e (iv) desempenho (objetivos), propostas por Smith \& Lewis (2011) podem impulsionar o desenvolvimento das capacidades dinâmicas comportamentos, habilidades, rotinas, processos e mecanismos de aprendizagem e governança do conhecimento.

\section{CONSIDERAÇÕES FINAIS}

A contribuição teórica central de nosso artigo está na identificação, de forma inédita, de diferentes tensões organizacionais como impulsionadoras de capacidades dinâmicas no contexto de organizações híbridas. As proposições desenvolvidas e o modelo proposto indicam que devemos compreender, de forma análoga aos pressupostos da teoria estruturalista (Bastos \& Seidel, 1992), as tensões como potenciais oportunidades para diferentes tipos de empreendimentos, em especial para os negócios sociais abordados de forma teórica em nosso estudo.

Como limitação do estudo, destacamos a não operacionalização dos elementos componentes do modelo e a ausência de testes entre estas relações. Devido ao ineditismo destas relações, defendemos que pesquisas futuras são necessárias para a operacionalização e validação dos componentes deste modelo, também por meio do teste de suas proposições basilares. Outra oportunidade de pesquisa futura está no desenvolvimento de uma escala de mensuração de tensões e capacidades dinâmicas em organizações híbridas e, de forma mais ampla, em outras tipologias organizacionais.

Apesar da relevância mundial do tema de capacidades dinâmicas, verificamos que no Brasil ainda são relativamente baixos os números de estudos que lidem especificamente como fatores catalisadores de capacidades dinâmicas nas organizações. Sugerimos, portanto, que mais pesquisadores invistam seus esforços de pesquisa na investigação de como outros tipos de organizações híbridas lidam com seus desafios inerentes ao hibridismo. Sob esta ótica, também sugerimos estudos que investiguem o modo como as capacidades dinâmicas se desenvolvem e como elas se sustentam ao longo do tempo neste tipo de empreendimento. 


\section{REFERÊNCIAS}

Aragão, L. A., Forte, S. H. A. C., \& de Oliveira, O. V. (2010). Visão baseada em recursos e capacidades dinâmicas no contexto brasileiro: a produção e a evolução acadêmica em dez anos de contribuições. READ-Revista Eletrônica de Administração, 16(2), 376-396.

Andreeva, T. \& Chaika, V. (2006). Dynamic capabilities: what they need to be dynamic? [Working Paper, 10 (E)] St. Petersburg State University, São Petersburgo.

Barney, J. (1991). Firm resources and sustained competitive advantage. Journal of Management, 17(1), 99-120.

Barreto, I. (2010). Dynamic capabilities: A review of past research and an agenda for the future. Journal of Management, 36(1), 256-280.

Bastos, A. V. B., \& Seidel, T. T. (1992). O conflito nas organizações: a trajetória de sua abordagem pelas teorias organizacionais. Revista de Administração (São Paulo), 27(3), 48-60.

Battilana, J., \& Dorado, S. (2010). Building sustainable hybrid organizations: the case of commercial microfinance organizations. Academy of Management Journal, 53(6), 1419-1440.

Bygdas, A. L. (2006, July). Enacting dynamic capabilities in distributed organisational environments. Proceedings of the EGOS Conference, Bergen, Norway, 22.

Collis, D. J. (1994). Research note: how valuable are organizational capabilities? Strategic Management Journal, 15(Suppl.), 143-152.

Danneels, E. (2011). Trying to become a different type of company: Dynamic capability at Smith Corona. Strategic Management Journal, 32(1), 1-31.

Dosi, G., Faillo, M., \& Marengo, L. (2008). Organizational capabilities, patterns of knowledge accumulation and governance structures in business firms: an introduction.

Eisenhardt, K. M., \& Martin, J. A. (2000). Dynamic capabilities: what are they? Strategic Management Journal, 21(10/11), 1105-1121.

Gerard, J. A. A (2009). Theory of organizational routines: development of a topology and identification of contextual determinants. Dissertation Thesis, UMI Dissertation Publishing, Ann Arbor, Michigan.
Grassl, W. (2012). Business models of social enterprise: A design approach to hybridity. ACRN Journal of Entrepreneurship Perspectives, 1(1), 37 60 .

Helfat, C. E., Finkelstein, S., Mitchell, W., Peteraf, M., Singh, H., Teece, D., \& Winter, S. G. (2009). Dynamic capabilities: Understanding strategic change in organizations. John Wiley \& Sons.

Iizuka, E. S., Varela, C. A., \& Larroude, E. R. A. (2015). Social business dilemmas in Brazil: Rede Asta case. Revista de Administração de Empresas, 55(4), 385-396.

Jäger, U. P., \& Schröer, A. (2014). Integrated organizational identity: A definition of hybrid organizations and a research agenda. VOLUNTAS: International Journal of Voluntary and Nonprofit Organizations, 25(5), 1281-1306.

Jay, J. (2013). Navigating paradox as a mechanism of change and innovation in hybrid organizations. Academy of Management Journal, 56(1), 137-159.

Lüscher, L. S., \& Lewis, M. W. (2008). Organizational change and managerial sensemaking: Working through paradox. Academy of Management Journal, 51(2), 221-240.

McKelvie, A., \& Davidsson, P. (2009). From resource base to dynamic capabilities: an investigation of new firms. British Journal of Management, 20(1), 63-80.

Meirelles, D.S., \& Camargo, Á. A. B. (2014). Capacidades Dinâmicas: o que são e como identificá-las?.Revista de Administração Contemporânea, 18(SPE), 41-64.

Michelini, L., \& Fiorentino, D. (2012). New business models for creating shared value. Social Responsibility Journal, 8(4), 561-577.

O'Reilly III, C. A., \& Tushman, M. L. (2008). Ambidexterity as a dynamic capability: Resolving the innovator's dilemma. Research in organizational behavior, 28, 185-206.

Pache, A. C., \& Santos, F. (2013). Inside the hybrid organization: Selective coupling as a response to competing institutional logics. Academy of Management Journal, 56(4), 972-1001.

Penrose, E. T. (1959). The theory of the growth ofthe firm. New York: Sharpe. 
Peteraf, M., Di Stefano, G., \& Verona, G. (2013). The elephant in the room of dynamic capabilities: Bringing two diverging conversations together. Strategic Management Journal, 34(12), 1389-1410.

Schumpeter, J. A. (1934). The theory of economic development. Cambridge. MA: Harvard.

Smith, W. K., \& Lewis, M. W. (2011). Toward a theory of paradox: A dynamic equilibrium model of organizing. Academy of Management Review, 36(2), 381-403.

Smith, W. K., Gonin, M., \& Besharov, M. L. (2013). Managing social-business tensions: A review and research agenda for social enterprise. Business Ethics Quarterly, 23(3), 407-442.

Teece, D. J., Pisano., G., \& Shuen, A. (1997.). Dynamic capabilities and strategic management. Strategic Management Journal, 18(7), 509-533.

Teece, D. J. (2007). Explicating dynamic capabilities: the nature and microfoundations of (sustainable) enterprise performance. Strategic Management Journal, 28(13), 1319-1350.

Teece, D. J. (2009). Dynamic capabilities \& strategic management. Oxford: Oxford University Press.

Teece, D. J. (2012). Dynamic capabilities: Routines versus entrepreneurial action. Journal of Management Studies, 49(8), 1395-1401.
Vogel, R., \& Güttel, W. H. (2013). The dynamic capability view in strategic management: A bibliometric review. International Journal of Management Reviews, 15(4), 426-446.

Walchhutter, S., Romani-Dias, M., \& Barbosa, A. S. (2016). Negócios sociais: um olhar sobre tensões e dilemas no contexto brasileiro. Anais do XIX Seminários em Administração, São Paulo, SP, Brasil, 19.

Wang, C. L., \& Ahmed, P. K. (2007). Dynamic capabilities: a review and research agenda. International Journal of Management Reviews, 9(1), 31-51.

Wilden, R., Devinney, T. M., \& Dowling, G. R. (2016). The architecture of dynamic capability research identifying the building blocks of a configurational approach. Academy of Management Annals, 10(1), 997-1076.

Williamson, O. E. (1975). Markets and hierarchies: Analysis and antitrust implications - a study in the economics of internal organizations. New York: Free Press, Macmillan.

Winter, S. G. (2003). Understanding dynamic capabilities. Strategic Management Journal, 24(10), 991-995.

Zollo, M., \& Winter, S. G. (2002). Deliberate learning and the evolution of dynamic capabilities. Organization $\quad$ Science, 13(3),339-351. 